\title{
Zinc Vacancy-Induced Room-Temperature Ferromagnetism in Undoped ZnO Thin Films
}

\author{
Hongtao Ren, ${ }^{1}$ Gang Xiang, ${ }^{1}$ Gangxu Gu, ${ }^{1}$ Xi Zhang, ${ }^{1}$ Wenjun Wang, ${ }^{2}$ Peng Zhang, \\ Baoyi Wang, ${ }^{3}$ and Xingzhong $\mathrm{Cao}^{3}$ \\ ${ }^{1}$ Department of Physics and Key Laboratory for Radiation Physics and Technology of Ministry of Education, Sichuan University,
Chengdu 610064, China
${ }^{2}$ Institute of Physics and Center for Condensed Matter Physics, Chinese Academy of Sciences, Beijing 100080, China
${ }^{3}$ Key Laboratory of Nuclear Analytical Techniques, Institute of High Energy Physics, Chinese Academy of Sciences,
Beijing 100049, China
}

Correspondence should be addressed to Gang Xiang, gxiang@scu.edu.cn and Xi Zhang, xizhang@scu.edu.cn

Received 29 June 2012; Accepted 19 July 2012

Academic Editor: Weichang Hao

Copyright (C) 2012 Hongtao Ren et al. This is an open access article distributed under the Creative Commons Attribution License, which permits unrestricted use, distribution, and reproduction in any medium, provided the original work is properly cited.

Undoped $\mathrm{ZnO}$ thin films are prepared by polymer-assisted deposition (PAD) and treated by postannealing at different temperatures in oxygen or forming gases $\left(95 \% \mathrm{Ar}+5 \% \mathrm{H}_{2}\right)$. All the samples exhibit ferromagnetism at room temperature (RT). SQUID and positron annihilation measurements show that post-annealing treatments greatly enhance the magnetizations in undoped $\mathrm{ZnO}$ samples, and there is a positive correlation between the magnetization and zinc vacancies in the $\mathrm{ZnO}$ thin films. XPS measurements indicate that annealing also induces oxygen vacancies that have no direct relationship with ferromagnetism. Further analysis of the results suggests that the ferromagnetism in undoped $\mathrm{ZnO}$ is induced by $\mathrm{Zn}$ vacancies.

\section{Introduction}

The dilute magnetic semiconductors (DMSs) have attracted increasing attention due to the potential applications in spintronic devices in recent years $[1,2]$. Since the roomtemperature (RT) ferromagnetism in Co-doped $\mathrm{ZnO}$ has been predicted by Dietl et al. with the Zener model of ferromagnetism [3], extensive theoretical and experimental studies have been performed on $\mathrm{ZnO}$ [4-12]. Various mechanisms, such as intrinsic defects [4-6] (zinc vacancy $\left(\mathrm{V}_{\mathrm{Zn}}\right)$, oxygen vacancy $\left(\mathrm{V}_{\mathrm{O}}\right)$, etc. $)$, extrinsic defects [7-10] (TM and non-TM dopants), and the correlation of intrinsic and extrinsic defects $[11,12]$ have been declared to induce the room-temperature (RT) ferromagnetism in $\mathrm{ZnO}$. However, magnetic properties of $\mathrm{ZnO}$ still remain a heavily controversial issue: the theoretical and experimental results were often found contradictory to each other; the origin of ferromagnetism and the influence of the complex interactions between different defects on magnetic properties of $\mathrm{ZnO}$ are still not well understood. Among them, the ferromagnetism induced by intrinsic defects, such as $\mathrm{Zn}$ vacancies and oxygen vacancies, and the correlation between the magnetic properties and intrinsic defects are of particular interest, since intrinsic-defects-induced mechanism may suggest new pathways for preparing $\mathrm{ZnO}$-based spintronic devices and the physics of the simple system without dopants may be more straightforward.

In this paper, we systematically investigated the correlation between the magnetic properties and the intrinsic defects in undoped $\mathrm{ZnO}$. Undoped $\mathrm{ZnO}$ thin films were first grown on (0001) sapphire $\left(\mathrm{Al}_{2} \mathrm{O}_{3}\right)$ substrates by polymerassisted deposition (PAD) $[13,14]$ and then treated by postannealing at different temperatures in different atmospheres. We then measured the structures and the magnetic properties of the $\mathrm{ZnO}$ thin films.

The crystalline structure of the films was characterized by using X-ray diffractometer (XRD) with $\mathrm{Cu} \mathrm{K} \alpha$ radiation. The morphology of the samples was observed by a field emission scanning electronic microscopy (SEM). Magnetic measurements were performed using superconducting 


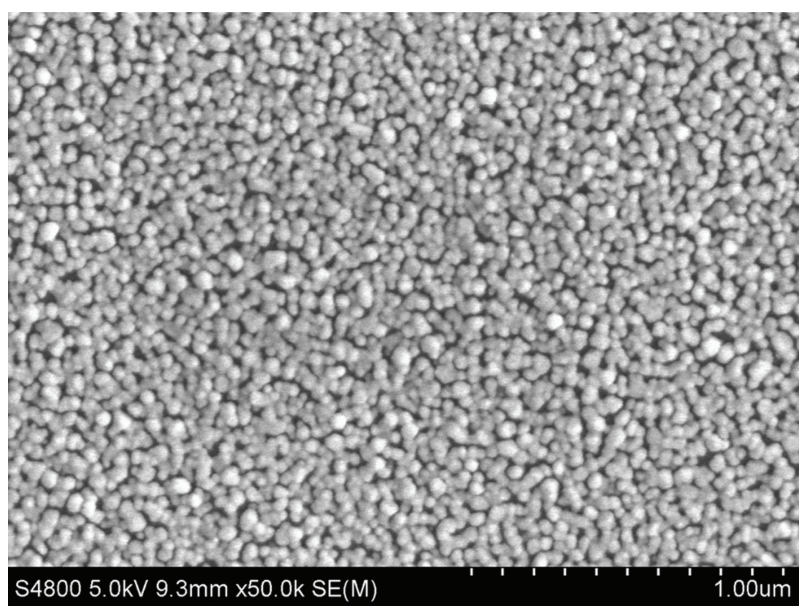

(a)

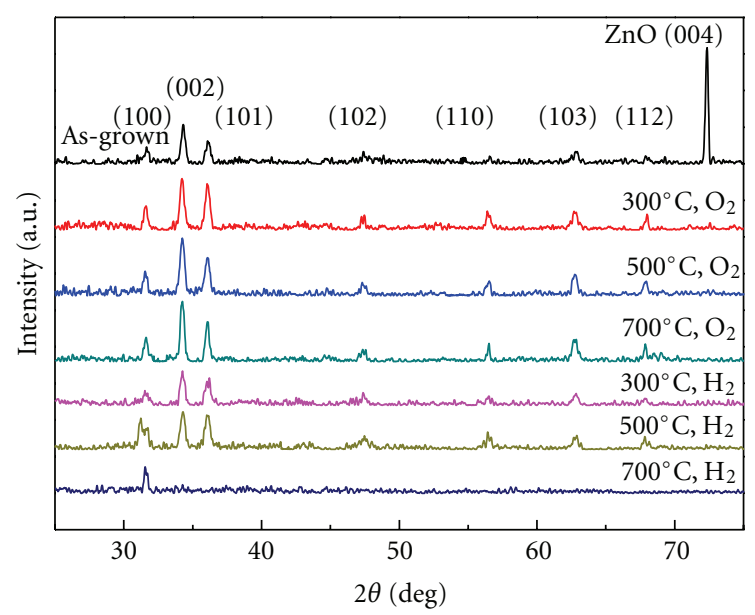

(b)

FIGURE 1: (a) SEM of nanocrystalline $\mathrm{ZnO}$ thin films (b) $\mathrm{XRD}$ spectra of $\mathrm{ZnO}$ thin films: as-grown, annealed at different temperatures in $\mathrm{O}_{2}$ or $\mathrm{H}_{2}$ atmosphere.

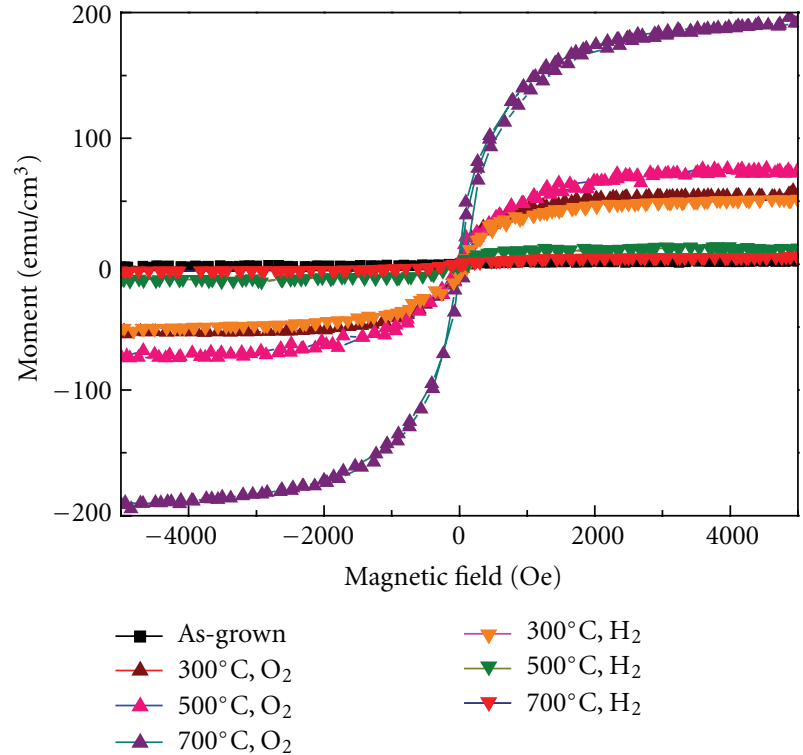

Figure 2: (a) $\mathrm{M}$ versus $\mathrm{H}$ of undoped $\mathrm{ZnO}$ thin films before and after annealing at different temperatures in $\mathrm{H}_{2}$ gas. (b) $\mathrm{M}$ versus $\mathrm{H}$ of undoped $\mathrm{ZnO}$ thin films before and after annealing at different temperatures in $\mathrm{O}_{2}$ gas.

quantum interference device (SQUID) magnetometer with the magnetic field applied parallel to the film surface at $300 \mathrm{~K}$. The chemical state of oxygen was measured by X-ray photoelectron spectroscopy (XPS). Cation vacancies were detected by positron annihilation spectroscopy (PAS).

\section{Experimental}

$\mathrm{ZnO}$ thin films were prepared by PAD of Zinc nitrate hexahydrate (purity $\geq 99.0 \%$ ) and ethylene imine polymer (purity $=99 \%$ ). To prepare Zn-polymer solution, $2.5 \mathrm{~g}$ zinc nitrate hexahydrate was dissolved in $40 \mathrm{ml}$ deionized water, and then $2 \mathrm{~g}$ ethylene imine polymer was added into the mixed solution. After stirring, the solution was purified by Amicon Ultra Centrifugal Filters with a 10,000 molar weightoff membrane. The solution was spin coated on (0001) Rcut sapphire substrates at the rates of $\sim 600 \mathrm{rpm}$ for $18 \mathrm{~s}$ and $\sim 2600 \mathrm{rpm}$ for $30 \mathrm{~s}$. The resultant coatings were gradually heated from room temperature to $500^{\circ} \mathrm{C}$ and stayed at that temperature for a period of about $1 \mathrm{~h}$ to burn out the polymer under an oxygen atmosphere. These as-grown films, which served as the precursors, were put into a horizontal quartz tube furnace for 3 minutes of heating at $900^{\circ} \mathrm{C}$. The heating and cooling rates were $75^{\circ} \mathrm{C} / \mathrm{s}$ for all the cases. After deposition, the samples were treated by post-annealing in oxygen gas or forming gas of $5 \% \mathrm{H}_{2}$ and $95 \%$ Ar gases (we use $\mathrm{H}_{2}$ to denote the forming gas for simplicity later) at 300,500 and $700^{\circ} \mathrm{C}$ for 30 minutes.

\section{Results and Discussions}

We first measured the structure and morphology of the $\mathrm{ZnO}$ samples. Figure 1(a) shows that the prepared as-grown samples are nanocrystalline $\mathrm{ZnO}$ thin films. The average crystallite size of the samples is about $50 \mathrm{~nm}$ and the film thickness is about $100 \mathrm{~nm}$. No crack or obvious protuberance can be observed within the visual field. As shown in Figure 2(b), XRD results indicate that all the thin films are polycrystalline wurtzite-type $\mathrm{ZnO}$ and no second peaks can be observed. After post-annealing, the (004) main peak in as-grown sample disappears, indicating formation of more defects in $\mathrm{ZnO}$ samples. The diffraction peaks of samples annealed in $\mathrm{O}_{2}$ at different temperatures are strong and similar to each other, while those of samples annealed in $\mathrm{H}_{2}$ at $700^{\circ} \mathrm{C}$ get much weaker, indicating the deteriorated quality of the sample. This is probably because $\mathrm{H}_{2}$ is a reducing gas and taking more amounts of oxygen atoms away from the sample at higher temperatures. 

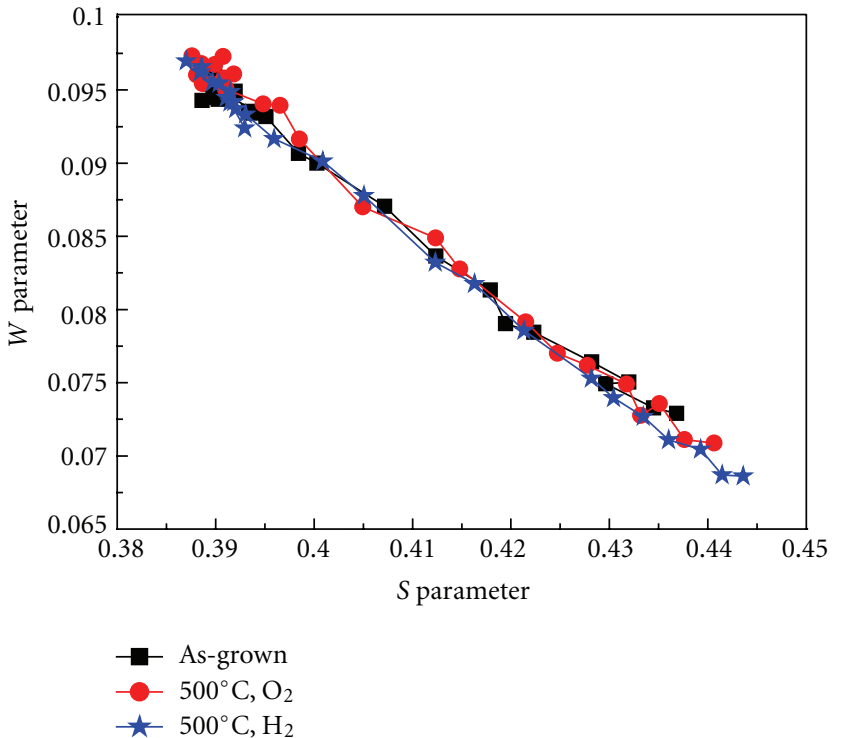
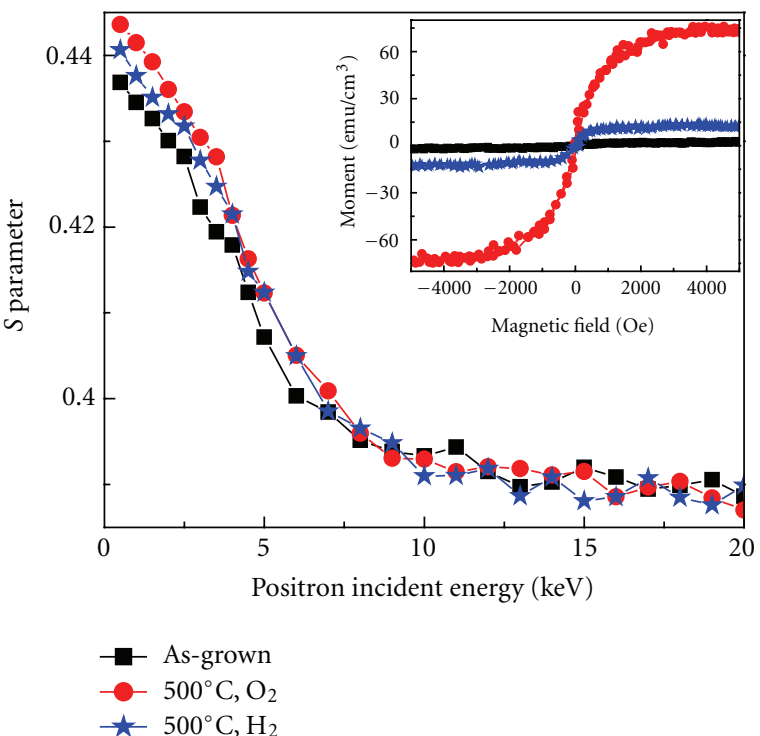

(a)

(b)

Figure 3: (a) Doppler-broadening parameter " $S$ " as a function of incident positron energy for the ZnO samples: as-grown, annealed at $500^{\circ} \mathrm{C}$ in $\mathrm{O}_{2}$ and annealed at $500^{\circ} \mathrm{C}$ in $\mathrm{H}_{2}$ atmosphere. (b) The spectra of $W$ parameter versus $S$ parameter.

The magnetization of the undoped $\mathrm{ZnO}$ thin films was measured by SQUID at room temperature, as shown in Figure 2. All the samples exhibit a clear hysteresis loop, indicating that these films are ferromagnetic at RT. The asgrown undoped sample shows weak ferromagnetism (M $\left.2.5 \mathrm{emu} / \mathrm{cm}^{3}\right)$. There are reports indicating that surface defects of the oxide thin film could cause ferromagnetism $[15,16]$. After post-annealing treatment at different temperatures in $\mathrm{O}_{2}$ or $\mathrm{H}_{2}$ gas, ferromagnetic ordering of all the samples was improved. As shown in Figure 2, in the case of annealing in $\mathrm{O}_{2}$ gas, the measured magnetization increases as annealing temperature increases, while in the case of annealing in $\mathrm{H}_{2}$ gas, the magnetization decreases as annealing temperature increases. However, the annealing effect at the same temperature in $\mathrm{O}_{2}$ atmosphere is always stronger than that in $\mathrm{H}_{2}$ in terms of enhancement of ferromagnetism in undoped $\mathrm{ZnO}$ thin films. Since annealing in oxygen atmosphere tends to prevent the formation of oxygen vacancies but promote the formation of $\mathrm{Zn}$ vacancies while annealing in hydrogen the opposite, the results above suggest that the enhancement of ferromagnetism of undoped $\mathrm{ZnO}$ thin films is related to the $\mathrm{Zn}$ vacancies in the sample.

In order to confirm the Zinc vacancies concentration, we carried out the positron annihilation analysis (PAS) measurements for a series of samples: as-grown, annealed at $500^{\circ} \mathrm{C}$ in $\mathrm{O}_{2}$ gas and annealed at $500^{\circ} \mathrm{C}$ in $\mathrm{H}_{2}$ gas. As shown in Figure 3(a), the linear $S$ - $W$ spectra suggest that there is only one type of cation vacancies, that is, zinc vacancies in the samples. The relationship between the $S$ values and positrons energy for the films is shown in Figure 3(b). The increase in the $S$ values in the annealed samples indicates that annealing introduces more $\mathrm{Zn}$ vacancies. In addition, the $S$ value in the sample annealed in $\mathrm{O}_{2}$ is bigger than that annealed in $\mathrm{H}_{2}$ at the same temperature. Since the magnetization of annealed samples increases and the magnetization of the samples annealed in $\mathrm{O}_{2}$ is bigger than that annealed in $\mathrm{H}_{2}$ at the same temperature, as shown in the insert of Figure 3(b), we see clearly positive correlation between the $\mathrm{Zn}$ vacancies and the magnetization of the undoped $\mathrm{ZnO}$ samples. In fact, theoretical studies show that the ferromagnetism can be induced by $\mathrm{Zn}$ vacancies instead of oxygen vacancies, in $\mathrm{ZnO}$ samples, and the magnetic moment mainly arises from the unpaired $2 p$ electrons at $O$ sites surrounding the $\mathrm{Zn}$ vacancies [17]. Therefore the ferromagnetic ordering can be enhanced by introducing more $\mathrm{Zn}$ vacancies.

On the other hand, we also investigate the variation of oxygen vacancies in the samples using XPS analysis. XPS O 1s scans of typical $\mathrm{ZnO}$ thin films are shown in Figures 4(a)-4(c). Two Gaussians were fitted to separate oxygen species. Then the profile can be fit by two symmetrical peaks, which are normally assigned as low-binding-energy component (LBEC) and high-binding-energy component (HBEC). Since the HBEC peak develops with increasing loss of oxygen, the development of the HBEC peak obviously leads to the asymmetry of the main peak. Changes in the ratios of HBEC/LBEC peak may result from the variations in the concentration of oxygen vacancies.

The variations of oxygen vacancies and the magnetization in the samples are shown in Figures $4(\mathrm{~d})-4(\mathrm{f})$. As shown in Figure 4(e), when annealing in $\mathrm{H}_{2}$, the magnetization decreases while oxygen concentration increases as annealing temperature increases. The negative correlation between the magnetization and the oxygen vacancies indicates that oxygen cannot be the origin of the ferromagnetism in these samples. In the case of annealing in hydrogen atmosphere, the oxygen vacancies are preferred, which results in higher 


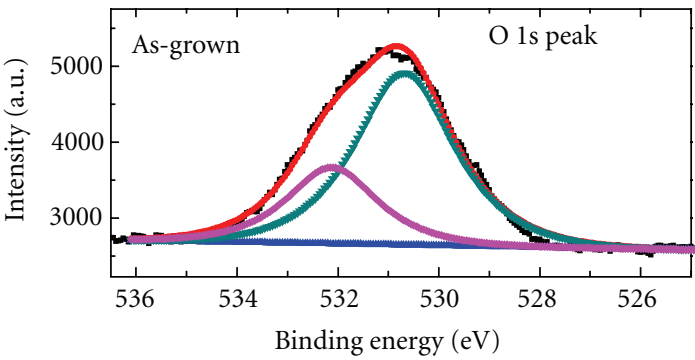

(a)

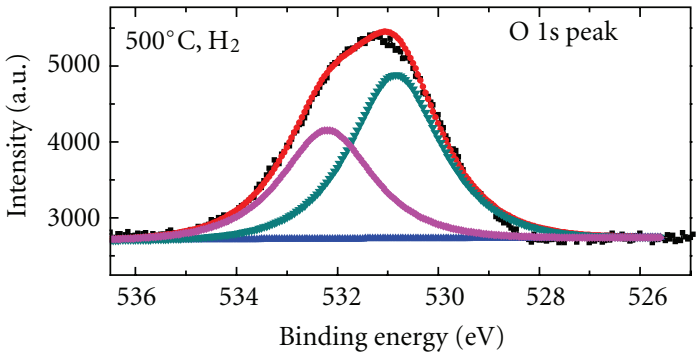

(b)

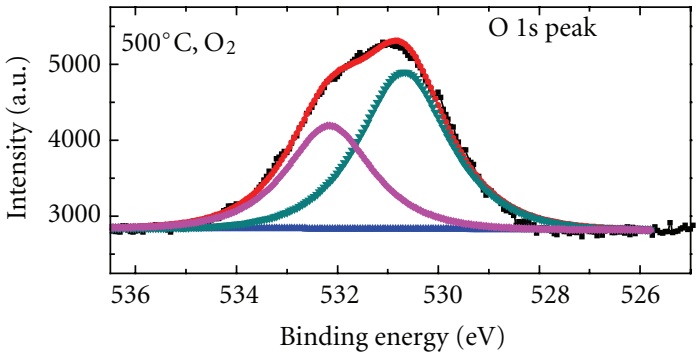

(c)

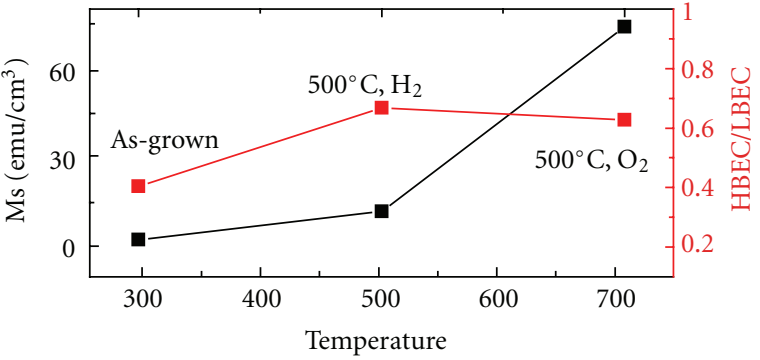

(d)

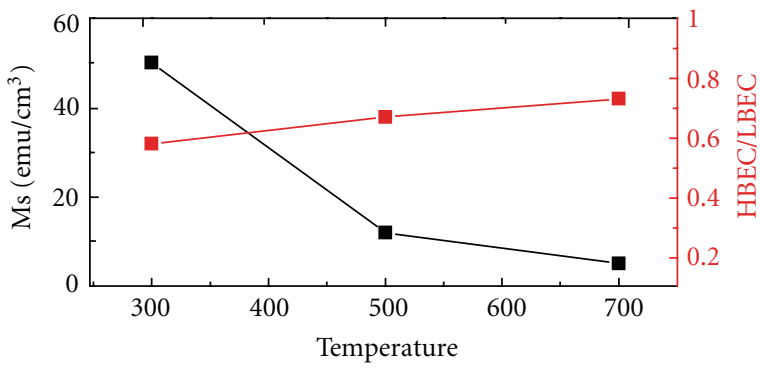

(e)

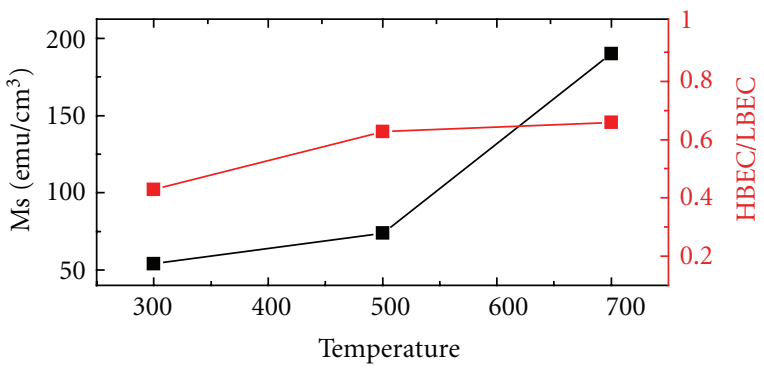

(f)

Figure 4: $(\mathrm{a}-\mathrm{c})$ Normalized XPS $\mathrm{O}$ 1s scans of $\mathrm{ZnO}$ samples: as-grown, annealed at $500^{\circ} \mathrm{C}$ in $\mathrm{H}_{2}$ atmosphere, and annealed at $500^{\circ} \mathrm{C}$ in $\mathrm{O}_{2}$ atmosphere; (d) The ratio of HBEC/LBEC peak and magnetization of as-grown, annealed $\left(500^{\circ} \mathrm{C}+\mathrm{H}_{2}\right)$, and annealed $\left(500^{\circ} \mathrm{C}+\mathrm{O}_{2}\right) \mathrm{ZnO}$ samples; (e-f) Corresponding ratios of HBEC/LBEC peak and magnetizations of samples annealed at different temperatures in $\mathrm{H}_{2}$ and $\mathrm{O}_{2}$ atmosphere, respectively.

oxygen concentration than the case of annealing in oxygen atmosphere, as shown in Figure 4(d). Since the oxygen vacancies have no positive contribution to the ferromagnetism and the reducing $\mathrm{H}_{2}$ gas may result in deterioration of sample quality at high temperature, the magnetization decreases as the annealing temperature increases. As shown in Figure 4(f), when annealing in $\mathrm{O}_{2}$, both the magnetization and the oxygen vacancies increase as the annealing temperature increases, but the increasing rate of magnetization is higher than that of oxygen vacancies. This can be understood as follows: annealing in oxygen atmosphere promotes the formation of $\mathrm{Zn}$ vacancies, so $\mathrm{Zn}$ vacancies increase more rapidly than oxygen vacancies; since the ferromagnetism is induced by $\mathrm{Zn}$ vacancies, the magnetization also increase more rapidly than that of oxygen vacancies.

\section{Conclusions}

In summary, we have grown undoped $\mathrm{ZnO}$ thin films by polymer-assisted deposition. All the $\mathrm{ZnO}$ thin films show
RT ferromagnetism. The enhancement of magnetization is observed after the samples are annealed at different temperatures in $\mathrm{O}_{2}$ or $\mathrm{H}_{2}$ gas. The clearly positive correlation between the magnetization and $\mathrm{Zn}$ vacancies indicates that Zinc vacancies are the origin of the ferromagnetism. Our results may be useful for the design and fabrication of spintronic devices based on $\mathrm{ZnO}$ DMS without dopants.

\section{Acknowledgments}

This work was supported by the Natural Science Foundation of China (NSFC) through Grant nos. 11004141 and 11174212 and by the Program for New Century Excellent Talents in University (NCET) through Grant no. 11-0351.

\section{References}

[1] I. Žutić, J. Fabian, and S. D. Sarma, "Spintronics: fundamentals and applications," Reviews of Modern Physics, vol. 76, no. 2, pp. 323-410, 2004. 
[2] S. A. Wolf, D. D. Awschalom, R. A. Buhrman et al., "Spintronics: a spin-based electronics vision for the future," Science, vol. 294, no. 5546, pp. 1488-1495, 2001.

[3] T. Dietl, T. Dietl, H. Ohno, F. Matsukura, J. Cibert, and D. Ferrand, "Zener model description of ferromagnetism in zincblende magnetic semiconductors," Science, vol. 287, no. 5455, pp. 1019-1022, 2000.

[4] M. Khalid, M. Ziese, A. Setzer et al., "Defect-induced magnetic order in pure ZnO films," Physical Review B , vol. 80, no. 3, Article ID 035331, 2009.

[5] G. Xing, D. Wang, J. Yi et al., "Correlated $d^{0}$ ferromagnetism and photoluminescence in undoped $\mathrm{ZnO}$ nanowires," Applied Physics Letters, vol. 96, Article ID 112511, 3 pages, 2010.

[6] P. Zhan, W. Wang, C. Liu et al., "Oxygen vacancy-induced ferromagnetism in un-doped $\mathrm{ZnO}$ thin films," Journal of Applied Physics, vol. 111, no. 3, Article ID 033501, 5 pages, 2012.

[7] K. Ueda, H. Tabata, and T. Kawai, "Magnetic and electric properties of transition-metal-doped $\mathrm{ZnO}$ films," Applied Physics Letters, vol. 79, no. 7, pp. 988-990, 2001.

[8] J. M. D. Coey, M. Venkatesan, and C. B. Fitzgerald, "Donor impurity band exchange in dilute ferromagnetic oxides," Nature Materials, vol. 4, no. 2, pp. 173-179, 2005.

[9] P. Sati, C. Deparis, C. Morhain, S. Schäfer, and A. Stepanov, "Antiferromagnetic interactions in single crystalline $\mathrm{Zn}_{1-x} \mathrm{Co}_{x} \mathrm{O}$ thin films," Physical Review Letters, vol. 98, no. 13, Article ID 137204, 4 pages, 2007.

[10] G. Gu, G. Xiang, and J. Luo, "Magnetism in transition-metaldoped ZnO: a first-principles study," Journal of Applied Physics, vol. 112, no. 2, Article ID 023913, 5 pages, 2012.

[11] J. B. Yi, C. C. Lim, G. Z. Xing et al., "Ferromagnetism in dilute magnetic semiconductors through defect engineering: Li-Doped ZnO," Physical Review Letters, vol. 104, no. 13, Article ID 137201, 4 pages, 2010.

[12] J.-J. Li, W.-C. Hao, H.-Z. Xu, and T.-M. Wang, "Variation of structural and magnetic properties with Co-doping in $\mathrm{ZN}_{1-x} \mathrm{Co}_{x} \mathrm{O}$ nanocrystals," Journal of Applied Physics, vol. 105, no. 5, Article ID 053907, 2009.

[13] Q. X. Jia, T. M. McCleskey, A. K. Burrell et al., "Polymerassisted deposition of metal-oxide films," Nature Materials, vol. 3, no. 8, pp. 529-532, 2004.

[14] A. K. Burrell, T. Mark McCleskey, and Q. X. Jia, "Polymer assisted deposition," Chemical Communications, no. 11, pp. 1271-1277, 2008.

[15] N. Sanchez, S. Gallego, and M. C. Muñoz, "Magnetic states at the oxygen surfaces of $\mathrm{ZnO}$ and Co-doped $\mathrm{ZnO}$," Physical Review Letters, vol. 101, no. 6, Article ID 067206, 2008.

[16] S. M. M. Yee, D. A. Crandles, and L. V. Goncharova, "Ferromagnetism on the unpolished surfaces of single crystal metal oxide substrates," Journal of Applied Physics, vol. 110, no. 3, Article ID 033906, 6 pages, 2011.

[17] Q. Wang, Q. Sun, G. Chen, Y. Kawazoe, and P. Jena, "Vacancyinduced magnetism in $\mathrm{ZnO}$ thin films and nanowires," Physical Review B, vol. 77, no. 20, Article ID 205411, 7 pages, 2008. 

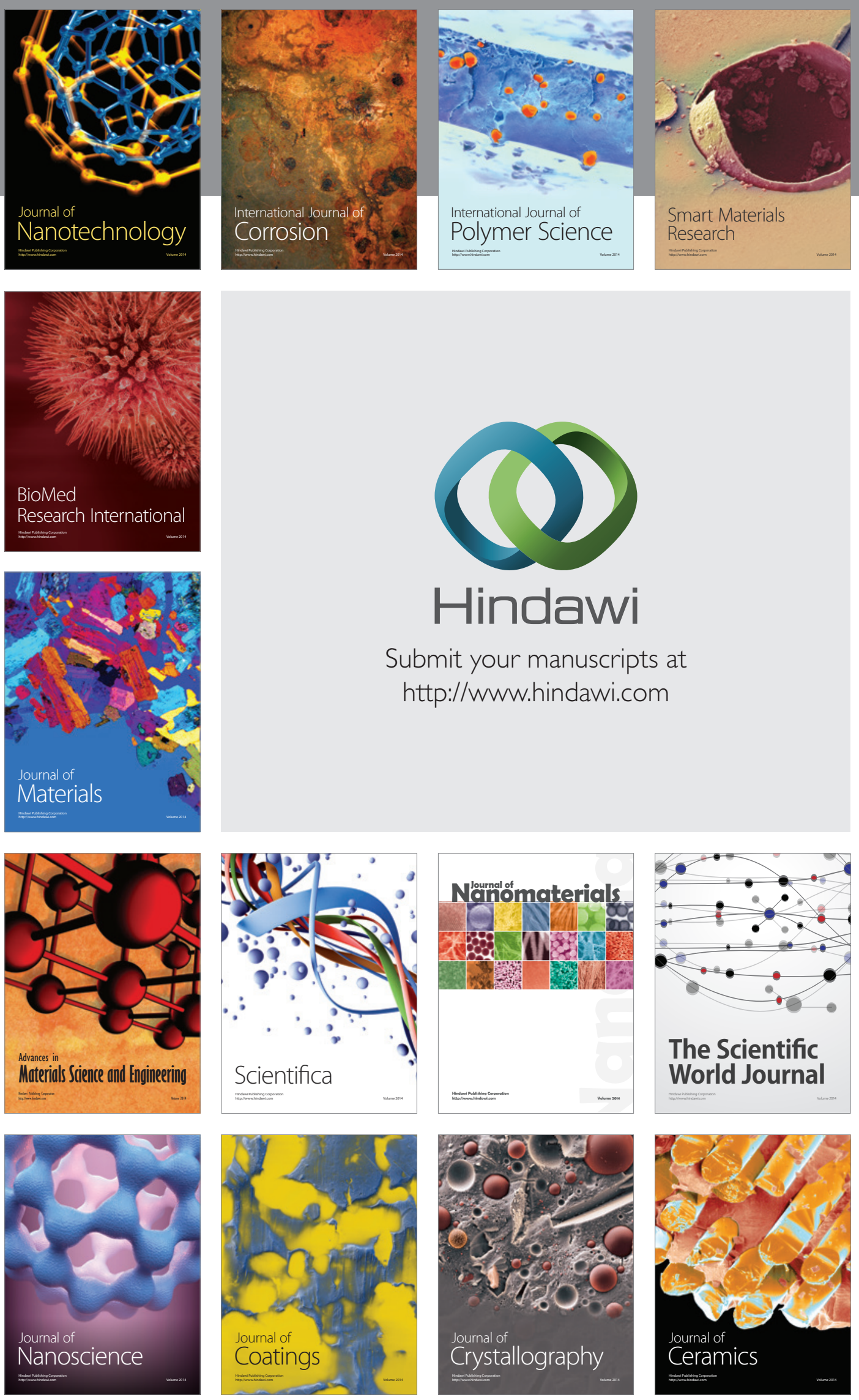

The Scientific World Journal

Submit your manuscripts at

http://www.hindawi.com

\section{World Journal}

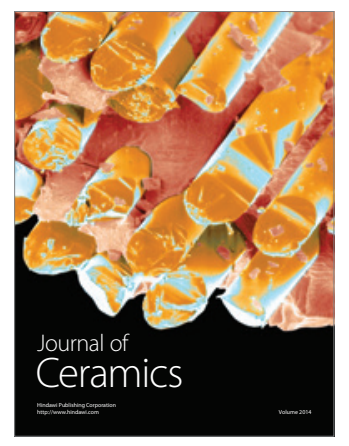

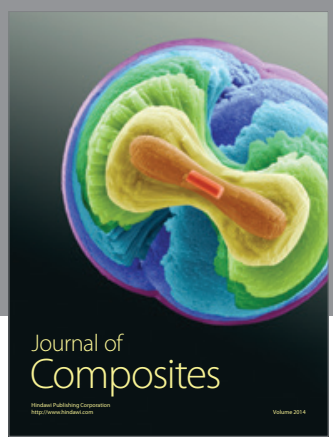
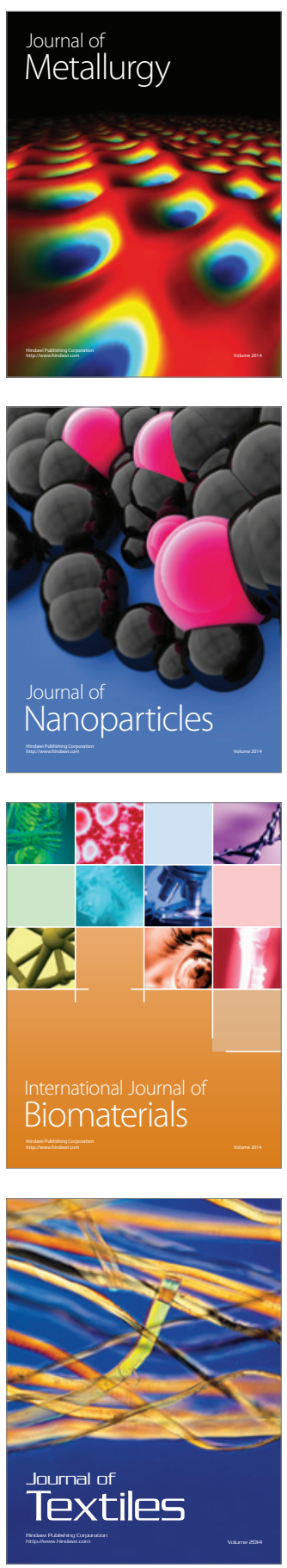